\title{
2014 Colorado alphaherpesvirus latency symposium
}

\author{
Randall J. Cohrs • Don Gilden
}

Received: 25 September 2014 / Accepted: 26 September 2014 / Published online: 5 November 2014

(C) Journal of NeuroVirology, Inc. 2014

\section{Introduction}

The fourth symposium of the Colorado Alphaherpesvirus Latency Society (CALS) convened May 14-16, 2014 at the Christiania Lodge in the relaxed setting of the quiet mountain community of Vail, Colorado. In attendance were 65 investigators (Fig. 1) who traveled over 128,000 miles to attend the 2-day symposium consisting of 28 oral and 16 poster presentations that discussed current advances in alphaherpesvirus latency research involving herpes simplex virus types 1 and 2, varicella zoster virus, bovine herpesvirus, Marek's disease virus, and pseudorabies virus. This year's plenary talk by Michael Stark, Brigham Young University, on the developing peripheral nervous system continued the CALS theme of a major presentation from an expert outside the field of virology to help view latency in a different light. This year also brought expansion of the mission of CALS to include mentorship of postdoctoral fellows as well as a new open forum to discuss pressing topics not previously addressed. Overall, 2014 marks the year in which CALS not only continued its founding mission of providing a venue to discuss new data, propose future experiments, and establish new collaborations while strengthening existing ones, but also included mentoring postdoctoral fellows and graduate students to ensure that the next generation of clinical/basic scientists investigating alphaherpesvirus latency benefits from the shared knowledge acquired by established investigators. A brief summary of each presentation follows.

R. J. Cohrs $(\bowtie) \cdot$ D. Gilden

Departments of Neurology and Microbiology \& Immunology,

University of Colorado School of Medicine, 12700 E. 19th Avenue,

Box B-182, Aurora, CO 80045, USA

e-mail: randall.cohrs@ucdenver.edu

\section{Reports}

Charles Grose, University of Iowa, presented evidence supporting the hypothesis that subclinical varicella zoster virus (VZV) reactivation boosts immunity to maintain virus latency and prevents zoster until late adulthood, as originally proposed by Hope-Simpson based on diagnosis and evaluation of 192 cases of zoster over a 16-year period. Obviously, very few cases of subclinical VZV reactivation have been rigorously documented in adults between the ages of 20 50 years because of the absence of rash. A case of VZV reactivation in a 23 -year-old otherwise healthy young man was described, in whom symptoms began suddenly with headaches and one seizure episode with no history of rash. MRI revealed a single small lesion in the left temporal lobe, and immunostaining of brain biopsy demonstrated VZV antigens. Sequence analysis of amplified VZV DNA revealed wild-type VZV. This case represents an episode of VZV reactivation in the brain without rash or radicular pain.

Erin Buckingham, University of Iowa, presented extensive studies of autophagy in VZV infection and showed that VZV induces autophagy in cultured cells and in cells from varicella and zoster skin vesicles. Immunofluorescence and confocal microscopy analyses of brain from a patient with zosterassociated encephalitis revealed cells that expressed VZV antigens as well as an autophagy marker. Identification of cell types in human brain infected with VZV after virus reactivation awaits further study.

Derek Jacobs, University of Florida, presented studies on the effect of the initial viral inoculum on the establishment of herpes simplex virus type 1 (HSV-1) latency in trigeminal ganglia cultures in a latency-associated transcript (LAT) manner. HSV-1 initially infects mucosal epithelial cells, after which virus undergoes multiple rounds of lytic infection before establishing lifelong latent infection in ganglionic neurons. No viral proteins are translated during latency, and viral 


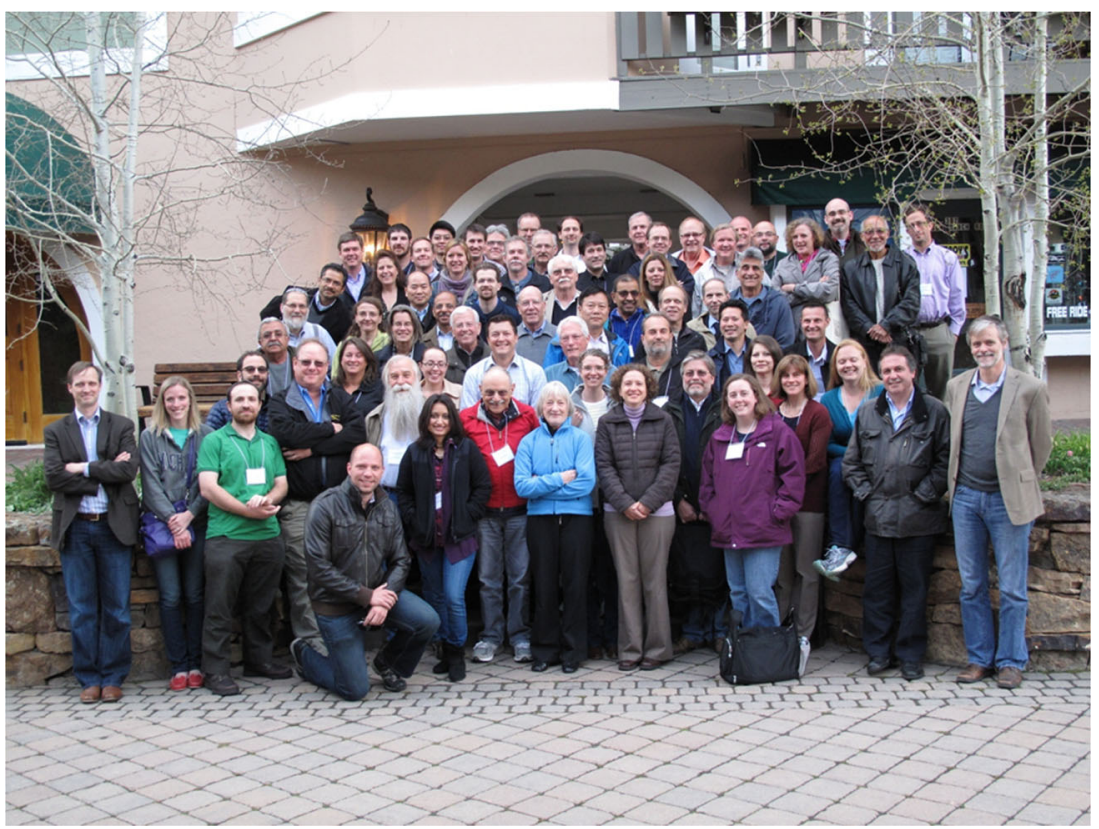

Fig. 1 Participants of the 2014 Colorado Alphaherpesvirus Latency Symposium. (left to right; row 1) Carter Cliff, Nikki Thellman, Amos Markus, Ian Mohr, Paul Kinchington, Daniel Depeldge, Randall Cohrs, Musarrat Farhana, Mike Gershon, Anne Gershon, Anna Cliffe, Ilhem Johnston, Karen Johnson, Stacey Efstathiou, and Luis Schang; (row 2) Homayon Ghiasi, Leigh Zerboni, Megan Steain, Cliff Ramsdell, Robert Hendricks, Angus Wilson, Chung Dang, and David Tscharke; (row 3) Ron Goldstein, Martine Aubert, Moriah Szpara, Satish Mehta, Don Messaoudi, Joel Rovnak, Erin Buckingham, Orkide Koyuncu, Christine

Gilden, Duane Pierson, Hua Zhu, Ravi Mahalingam, David Koelle, and Peter Kennedy; (row 4) Deepak Shukla, Andrea Bertke, Jason Chen, Derek Jacobs, Charles Grose, Donna Neumann, and Todd Margolis; (row 5) Michael Stark, Seth Frietze, Marieke Verweij, Ken Jones, John Blaho, David Davido, David Bloom, Judy Breuer, and Edouard Cantin; (row 6) Nicholas Baird, Chen Sherman, Travis Whitmer, Georges Verjans, Juergen Haas, Nigel Fraser, Benedikt Kaufer, Clinton Jones, Scott Schmid, Klaus Osterrieder, Joshua Geltz, Alexander Rowe, and Joshua Schiffer

transcription is restricted to a single locus, from which the major transcript is the non-coding LAT. The role of LAT in establishing latency is not fully understood but may be closely related to epigenetic remodeling of HSV-1 genomes. Jacob's laboratory found that infection of primary adult murine trigeminal ganglion cultures with increasing amounts of virus resulted in multiple phenotypes, with variable virus gene expression, virus DNA levels, epigenetic profiles, and reactivation efficiency. Virus transcript levels increased up to 10fold as a function of initial inoculum. Post-translational modifications of chromatin were also correspondingly dependent on initial inoculum: $\mathrm{H} 3 \mathrm{~K} 4 \mathrm{me} 3$ levels decreased up to 5-fold at higher multiplicity of infection (MOI), while H3K27me3 levels increased up to 10-fold. Strikingly, comparison of cultures infected with the parental $17+$ and the LAT promoter deletion $17 \Delta$ Pst showed that these phenotypes were LAT dependent, with $17 \Delta$ Pst exhibiting no significant change in either transcript levels or heterochromatic markers as a function of MOI. This suggests that LAT is a critical determinant of these dose-dependent phenotypes and that the initial HSV-1 inoculum is important in the establishment and maintenance of latency.

Luis Schang, University of Alberta, reported studies of mechanisms that activate transcription of latent HSV-1. Most current models propose that transcription is repressed during latency by silencing chromatin and that reactivation of virus transcription requires remodeling of chromatin, a notion supported by the observation that all histones are mobilized during lytic infection. Early mobilization that does not require viral proteins is consistent with cellular attempts to silence infecting viral genomes. An equivalent mobilization would silence the viral genomes to enter latency. Later enhanced mobilization, which requires viral proteins but not DNA replication, is consistent with desilencing of the viral genomes. Such mobilization would not occur during the establishment of latency but would be required for reactivation. The critical transcription activator ICP4 is known to play a major role in histone mobilization, based on studies showing that HSV-1 with a mutated ICP4 fails to mobilize histones H1.2, H2A, $\mathrm{H} 2 \mathrm{~B}, \mathrm{H} 3.1$, and $\mathrm{H} 3.3$ or transcription. Although this effect could be secondary to lack of early or late gene expression or DNA replication during infection with ICP4 mutants, transiently expressed ICP4 mobilized H1.2, H2B, H3.1, H3.3, and $\mathrm{H} 4$ in the absence of infection. These studies show that ICP4 mobilizes histones and raise the possibility that ICP4 expression during reactivation mobilizes histones away from previously latent genomes, facilitating transcription of all genes to complete reactivation.

Homayon Ghiasi, Cedars-Sinai Medical Center, presented studies of the effect of the interplay between LAT and the 
herpesvirus entry mediator (HVEM) on HSV-1 latency. To test whether viral entry receptors are involved in establishment of HSV-1 latency, LAT( + ) and LAT(-) viruses were used to evaluate the effect of LAT on HSV-1 receptors in ocularly infected mice. Latency was increased in trigeminal ganglia of wild-type mice infected with LAT(+) versus LAT(-) virus, and correlated with increased levels of HVEM, but not with other known HSV-1 receptors. Similarly, HVEM expression in vitro was upregulated in $\mathrm{C} 1300$ and Neuro2A cell lines stably expressing LAT. Finally, HVEM upregulation region of LAT was mapped to HSV-1 genomic nucleotides 119756119717 and 120145-120179. The results suggest that continuous upregulation of HVEM by LAT leads to more efficient binding of HSV-1 gD to HVEM in the latent microenvironment, thus enhancing HSV-1 latency and reactivation.

Clinton Jones, University of Nebraska, presented studies to identify factors that can stimulate productive virus infection during stress-induced reactivation from latency. These studies show that bovine herpesvirus 1 (BHV-1) establishes lifelong latent infection in sensory neurons after acute infection and that increased corticosteroid levels due to stress increase reactivation from latency. A single intravenous injection of dexamethasone induces reactivation in calves $100 \%$ of the time. Recent published studies demonstrated that within $1.5 \mathrm{~h}$ after treatment, bICP0 and a late protein (VP16) were consistently detected in a subset of trigeminal ganglionic neurons. Neurons expressing bICP0 also expressed VP16 and the glucocorticoid receptor. In contrast, other late viral proteins $(\mathrm{gC}$ and $\mathrm{gD})$ were not detected until $6 \mathrm{~h}$ after dexamethasone treatment. VP16+ trigeminal ganglionic neurons were detected $30 \mathrm{~min}$ after dexamethasone treatment of latently infected calves, indicating that VP16 is expressed early during dexamethasoneinduced reactivation from latency. The factor(s) that stimulates VP16 expression in sensory neurons during reactivation from latency remains to be identified, since none of the dexamethasone-induced cellular transcription factors previously identified in trigeminal ganglia transactivated the VP16 promoter. One such factor could be serum- and glucocorticoid-inducible kinase-1 (SGK1), which is highly stimulated by glucocorticoids and known to promote neuronal survival and neurite outgrowth. BHV-1 replication was strongly inhibited by the SGK1 inhibitor GSK 650394 in bovine kidney cells. SGK1 expression was induced during productive infection, further suggesting a role for SGK1 during productive infection. Overall, there is increasing evidence that dexamethasone, which simulates stress, has many effects on sensory neurons that ultimately stimulate viral gene expression and reactivation from latency.

Hua Zhu, Rutgers - New Jersey Medical School, presented data on factors critical for viral neuroinvasion. Neuronal involvement of VZV is of particular concern due to its long-term and severe sequelae after reactivation. This laboratory prepared a VZV open reading frame (ORF) deletion mutant and infected human epithelial cells, neurons, ex vivo skin, and dorsal root ganglia and SCID-hu mice. The screening approach identified ORF7 as a viral tegument protein required for efficient infection of both primary epithelial cells and cultured neurons, and revealed that ORF7 was dispensable for penetration of dendritic cells or T cells. Importantly, the ORF7 deletion mutant was incapable of infecting skin and dorsal root ganglia in vivo and is therefore an ideal vaccine candidate.

Amos Markus, Bar-Ilan University, described cellular transcriptome analysis revealing differential expression of proand anti-apoptosis genes by VZV-infected human neurons and fibroblasts. For these studies, this laboratory generated $>90 \%$-pure populations of neurons from human embryonic stem cells, infected them with green fluorescent protein (GFP)-expressing cell-free VZV, and used human wholegenome microarrays to compare their transcriptome to that of human foreskin fibroblasts infected in parallel. Based on a cutoff of a 2-fold change, transcription of 340 neuronal genes was significantly altered by VZV infection, including 223 changes unique to neurons. Gene ontology analysis revealed significantly increased transcription of genes in 17 functional categories in fibroblasts, while those in only one category were downregulated. Transcriptional changes in neurons were in eight functional categories that differed strikingly from those in foreskin fibroblasts. For example, neurons did not show the fibroblast-specific upregulation of gene groups classified as innate immune responses, NF $\kappa B$-related, response to stress, and DNA repair. The most striking transcriptional response difference between neurons and fibroblasts was in apoptosis-related genes: A large cluster of 193 pro-apoptotic genes was upregulated in fibroblasts, whereas no such clusters were upregulated in VZV-infected neurons, which instead showed upregulation of a cluster of 17 genes, including Trail-R4, BIRC5 (SURVIVIN), and BAG3, involved in antiapoptotic pathways. These changes are reflected in the observations that neurons productively infected with VZV do not undergo apoptosis in vitro as opposed to $\mathrm{VZV}$-infected nonneuronal cells. These data can serve as a basis for discovery of differences in virus-host interactions between these VZV target cell phenotypes.

Ronald Goldstein, Bar-Ilan University, discussed a model of VZV latency and reactivation based on neurons derived from human embryonic stem cells (hESCs). In initial studies of VZV infection of hESC-derived neurons, large numbers of infecting cells or high doses of cell-free virus resulted in productive infection. Others have reported non-productive infection of human stem cell derived neurons after exposure to a lower MOI of VZV. Based on those observations and on the dissociated murine sympathetic ganglion neuron model of HSV-1 latency in vitro, this laboratory developed an experimental system for studying silent and persistent infection by VZV that can be "reactivated" after many weeks. When 
hESC-derived neurons were exposed to cell-free VZV at low PFU in the presence of acyclovir, about $50 \%$ of culture wells did not display GFP fluorescence for up to 7 weeks. Quantitative PCR analysis revealed both VZV DNA and transcripts at 2, 4, and 7 weeks postinfection, with levels decreasing thereafter. Importantly, maintenance of silent infection for weeks after VZV infection does not require the continuous presence of acyclovir. Several methods were then used to attempt to reactivate VZV. Inhibition of HDAC or removal of NGF at 2-7 weeks after initial exposure to virus resulted in display of several GFP-fluorescent neurons in up to $30 \%$ of the previously GFP-negative neuronal wells. Quantitative PCR revealed large increases in $\mathrm{gB}$ and IE63 RNA after either treatment, confirming activation of VZV transcription. Other treatments, including superinfection with HSV, did not lead to VZV reactivation. Most recently, this laboratory has established a robust "reactivation" stimulus which induces large clusters of persistently infected neurons to express GFP. This new model may allow discovery of mechanisms underlying VZV latent infection and reactivation.

Andrea Bertke, Virginia Tech, described differences between HSV-1 and HSV-2 infection of autonomic ganglia after ocular infection of guinea pigs. Although HSV-1 and HSV-2 establish latency and reactivate from trigeminal ganglia (TG) in humans and animal models, viral DNA is also found in autonomic ganglia, including the sympathetic superior cervical ganglia (SCG) and parasympathetic ciliary (CG), pterygopalatine, submandibular, and otic ganglia. After ocular infection of guinea pigs, HSV-1 and HSV-2 viral DNAs were found in the TG, SCG, and CG at comparable levels. However, HSV-1 only expressed thymidine kinase transiently in SCG at 2 days postinfection, while HSV-2 expressed the enzyme throughout acute infection. In CG, HSV-2 expressed higher levels of thymidine kinase than did HSV-1. Both viruses expressed LAT in TG and SCG, but no HSV-2 LAT was detected in CG. HSV-1 and HSV-2 both reactivated ex vivo from a greater number of CG and SCG neurons compared to TG neurons, and HSV-1 cumulatively reactivated more efficiently from CG and SCG neurons compared to HSV-2 during the first 3 days in culture. In cultured neurons, HSV-1 and HSV-2 preferentially infected SCG neurons expressing adrenergic and glucocorticoid receptors, but HSV-1 did not demonstrate this preference in TG neurons. HSV-2 productive infection was limited in TG neurons expressing glucocorticoid and adrenergic-betal receptor, suggesting that epinephrine and cortisol may have different effects on HSV-1 and HSV-2 in sensory versus autonomic neurons. These findings suggest that differences in viral gene expression in autonomic ganglia contribute to differences between HSV-1 and HSV-2 in the preferred anatomical site of reactivation.

Daniel Depledge, University College London, discussed genetic correlates of VZV encephalitis and tropism in different body compartments. After primary infection, VZV becomes latent and has the potential to reactivate with waning immunity. VZV encephalitis, while infrequent as compared to its HSV-1 counterpart, is linked to morbidity and mortality. VZV has previously been isolated from numerous body compartments, including the skin, saliva, lungs, blood, and cerebrospinal fluid, but no attempts have been made to correlate viral genetics with tropism. Whole VZV genomes were captured and sequenced directly from 42 samples of clinical material noted above obtained from patients with varicella and zoster, including six patients with VZV encephalitis. Phylogenetic analyses confirmed clade-related clustering, with potentially two to three recombinant genomes present and several infections comprising a mix of genotypes. Sequence alignments show no fixed differences between encephalitis and nonencephalitis samples, and overall genetic diversities of each phenotypic group were comparable. In addition, VZVs sampled from multiple body compartments in the same patient were genetically identical, although vesicle populations were generally monomorphic compared to polymorphic populations from other body compartments. These differences were attributed to the presence of a stronger "bottleneck" effect when VZV infects vesicles than when virus moves between compartments. The most intriguing observation was a prevalence of ORF stop codons at frequencies $>10 \%$ in cerebrospinal fluid, where greater proportions of viral DNA may come from defective virus. Viral spread in the brain is highly cell-associated and up to six ORFs are not required for replication.

Angus Wilson, New York University School of Medicine, discussed the increasing appreciation of cell culture models as tools to study alphaherpesvirus latency and reactivation, and described studies using primary superior cervical ganglia neurons aimed at defining physiological parameters that determine whether de novo HSV-1 infections enter latency or sustain productive replication. By progressively lowering the virus to neuron ratio, latency was efficiently established without antiviral agents. Cultures remained healthy, with detectable LAT RNA and almost no expression of productive cycle markers. Cultures treated with agents that block NGF signaling or the host DNA damage response yielded infectious virus indicative of productive reactivation, and importantly, the viral gene expression profile showed the same biphasic (phase I-II) kinetics defined earlier using cultures established in the presence of acyclovir. Furthermore, microfluidic chamber devices permitted viral entry through axons rather than cell bodies, allowing establishment of latency with greater doses of virus. This route mimics natural infection in which HSV-1 first enters a nerve via axonal termini innervating peripheral tissue. These studies indicate that neuron intrinsic repression mechanisms are sufficient to establish HSV-1 latency and that viral reactivation undergoes two mechanistically distinct phases of transcription irrespective of earlier treatment with acyclovir. 
Moriah Szpara, Pennsylvania State University, described variation among strains of HSV-1. HSV-1 strains differ in virulence, establishment of latency, and frequency of reactivation. Variability exists in even a single strain of HSV-1 with mutations arising and generating new phenotypes. The extent to which this occurs in vivo is unknown. This group analyzed and quantified variations in plaque morphology in the classic HSV-1 F and KOS strains. As a model of intrastrain variability, syncytial variants of three strains were isolated and examined for their genetic relatedness using Illumina highthroughput sequencing and a new de novo assembly pipeline. All spontaneous syncytial variants shared an identical mutation in a core fusion protein, raising the possibility that hotspots for mutation exist in the HSV genome. The ability to rapidly sequence and assemble HSV genomes will allow exploration of when and how frequently new HSV variants arise in vivo, and how the pool of latent HSV genomes is affected by new HSV exposures.

David Tscharke, Australian National University, presented work that examined HSV activity during the establishment of latency in mice (5-30 days after infection) and at later times using a model in which $\beta$-galactosidase is constitutively expressed in cells with HSV-driven Cre recombinase expression. A panel of viruses with Cre under the control of various HSV promoters enabled counting of cells that showed different types of viral activity: Entry of a virus genome was examined by driving Cre from the cytomegalovirus immediate-early promoter (pCMV). Use of two lytic gene promoters, $\mathrm{gB}$ and ICP47, to track expression of these HSV genes showed that marked neurons and spread of virus to dorsal root ganglia distant from the infected dermatome on the flank continued to increase for 5-6 days beyond the time of peak infectious virus load. Results using viruses with Cre driven by pgB and pICP47 were consistent with lytic gene expression occurring in many dorsal root ganglia neurons during the establishment phase, suggesting that more virus activity is occurring at this time than had been appreciated. Finally, neurons of mice infected with viruses expressing Cre under pgB and pICP47, but not pCMV, continued to be marked during latency (day 40-100). This finding is the first demonstration that lytic promoter activity leads to protein production in latency, and provides evidence of an antigen source to drive $\mathrm{gB}$-specific $\mathrm{CD} 8+\mathrm{T}$ cell activation observed during latency.

Christine Johnston, University of Washington, presented data showing dual strain infection and simultaneous shedding of two HSV-2 strains from the genital tract. To estimate the prevalence of HSV-2 reinfection, DNA from human genital HSV-2 lesions containing $\geq 7 \log _{10}$ copies of HSV DNA/swab was submitted for Illumina next-generation sequencing (NGS). After subtracting human and highly repetitive sequences, reads were aligned to the reference HSV-2 genome (HG52). SAMtools software was used to identify sites of heterogeneity. PCR-Sanger sequencing of a variable locus was used to confirm selected NGS findings. Of 49 sequences from 39 individuals with adequate read depth, there was a median of 73.9-fold coverage (range 9.8- to 771-fold) of the HSV genome. Ten persons (three HIV-infected) had paired samples collected at a median of 2 years apart (range 0.116 years). Two (20\%) persons had paired sequences that differed at only one single-nucleotide polymorphism, and six $(60 \%)$ had identical sequences at two time points. Two $(20 \%)$ HIV-infected persons had sequences that differed at 186 single-nucleotide polymorphisms, consistent with dual strain infection. The third HIV-infected person had simultaneous dual strain shedding, with sequence heterogeneity at 211 sites within the index swab DNA; all of 11 additional rectal and genital specimens collected from this individual over a 13-day reactivation episode revealed both HSV-2 strains. These data suggest that distinct HSV-2 strains may simultaneously and continuously reactivate over several days, consistent with dual strain ganglionic infection. Different strains may also be detected in isolation in the same person over time. Persons without dual strain infection did not have detectable withinhost evolution, suggesting reactivation of archived HSV-2.

Nikki Thellman, Van Andel Research Institute, described an immortalized human dorsal root ganglia cell line that provides a novel context to study HSV reactivation. HSV-1 establishes lifelong latent infection in ganglia with occasional reactivation by mechanisms that are not well understood due, in part, to the difficulty in establishing valid and robust experimental models of latency. Recently, primary rodent neuronal cultures have emerged as an option for studying molecular mechanisms of HSV-1 reactivation in vitro. One complication of this approach arises from molecular differences in rodent host cell proteins known to associate with viral proteins. As an alternative, this laboratory proposes an immortalized human dorsal root ganglia cell line, HD10.6, which proliferates in a committed sensory neuronal progenitor state via a tetracycline-regulated v-myc oncogene. In the presence of doxycycline, HD10.6 cells terminally differentiate to exhibit neuronal morphology and express sensory-neuron-associated markers such as TrkA (nerve growth factor receptor) and perpherin. This laboratory is currently optimizing HSV-1 latent infection in this novel in vitro system, expecting it to allow studies of HSV-1 molecular reactivation from human ganglionic neurons. HD10.6 cells infected with HSV-1 strain DG1, which encodes a VP16-GFP fusion protein, were monitored for cessation of late lytic gene expression suggestive of latent infection, and for reactivation events as a result of various external stimuli (NGF withdrawal and heat shock). Consistent with the hallmarks of HSV latency, expression of the latency-associated transcript and absence of lytic gene expression was confirmed by quantitative RT-PCR.

Orkide Koyuncu, Princeton University, discussed outcome determinants of retrograde alphaherpesvirus infection in neurons. Alphaherpesviruses such as the human pathogen HSV-1 
and the animal pathogen pseudorabies virus (PRV) invade the peripheral nervous system through axonal termini, with establishment of latent infection in ganglia there dependent on the long-distance retrograde transport of viral capsids and tegument proteins to neuronal nuclei. Several viral proteins have been reported to interact with the dynein microtubule motors that catalyze transport, but the initial steps leading to efficient establishment of infection remain unknown. To define these critical initial events in alphaherpesvirus infection, this laboratory utilized a compartmented primary neuron culture system in which distal axons of superior cervical ganglionic (SCG) neurons are infected with fluorescent protein-tagged $\mathrm{PRV}$, retrograde capsid transport in axons is visualized, and progress of infection in cell bodies is monitored. Previous results showed that upon infection at high multiplicity (MOI 10-100), entering PRV particles induce and require local synthesis of several cytoskeletal, signaling, transport, and metabolic proteins in axons, and establish productive infection in the neuronal soma. After low-MOI infection of axons (MOI $\leq 1$ ), entry and trafficking of PRV particles are inefficient, and virus replication in SCG cell bodies is either delayed or "silent" as in quiescent infection. Addition of 100-fold more UV-inactivated or replication-deficient PRV particles did not restore replication of low-MOI PRV infection but did completely abolish replication of low-MOI PRV in SCG nuclei. These results suggest that early events in axonal herpesvirus infection determine efficiency of infection in ganglia. Moreover, there is not only a multiplicity threshold to initiate replication in the neuronal nucleus but also at least one limiting factor in axons for which entering capsids and tegument proteins compete.

Satish Mehta, NASA-Johnson Space Center-EASI, discussed a rapid, highly sensitive diagnostic assay for VZV DNA in the saliva of zoster patients, who often have severe complications from VZV reactivation such as postherpetic neuralgia, zoster paresis, cranial nerve palsies, myelitis, meningoencephalitis, vasculopathy, and multiple serious ocular disorders, all in the absence of rash, making diagnosis difficult. In such instances, virologic verification of disease produced by VZV relies on detection of VZV DNA or anti-VZV IgG antibody in cerebrospinal fluid or, less often, the presence of VZV DNA in blood mononuclear cells or anti-VZV IgM antibody in serum or cerebrospinal fluid. Because VZV DNA is present in saliva of patients with acute zoster, as well as in patients with zoster sine herpete, saliva may be helpful in diagnosing disease produced by VZV in the absence of rash. This laboratory developed a saliva-based assay to detect VZV DNA that provides a 3- to 9-fold increase in DNA yield in saliva collected by passive drool or synthetic swab. Centrifugation of saliva at high speed for 20 min reduces completion time for the assay from 6 to $1 \mathrm{~h}$.

Chung Dang, Fred Hutchinson Cancer Research Center, described the use of engineered nucleases to prevent HSV-1 reactivation from latently infected mouse ganglionic neurons. This laboratory tested whether engineered homing endonucleases (HEs) can disable latent HSV-1 in infected mouse trigeminal ganglion neurons. These HSV-specific HEs induce double-stranded DNA breaks within their target sequences located in HSV essential genes, UL19 or UL30, leading to mutations of HSV genomes as a result of error-prone nonhomologous end joining. This laboratory previously demonstrated that exposure to HEs specific for HSV-1 UL19, along with the 3 '-repair exonuclease 2 (Trex2), disrupts UL19 and inhibits virus production in an in vitro model of HSV latency. In a mouse model of HSV-1 latency developed by intradermal injection of the whisker pad with virus, latent HSV-1 was able to reactivate from explanted trigeminal ganglia at 30 days postinoculation, producing infectious virus as measured by plaque assay. Recombinant AAV8 vectors were then administered via the same whisker pad route, and in vivo transduction efficiency was quantified by evaluating AAV8 transgene expression in neuronal cultures of explanted trigeminal ganglia; transgene expression was detected as early as 5 days after infection, and by 30 days, $40 \%$ of neurons were transduced. Current studies include optimizing levels of in vivo transgene expression and the degree to which HE delivery by AAV vectors can disrupt latent HSV in vivo. Targeted mutagenesis of latent HSV genomes is an encouraging strategy to prevent HSV reactivation.

Kenneth Jones, University of Colorado, described VZV infection of human neurons silencing of cholesterol biosynthesis pathways. Analysis of the cellular transcriptome by next-generation RNA sequencing identified 304 neuronal genes that were differentially transcribed $(Q<0.05)$ in VZVinfected neurons compared to uninfected neurons. Pathway analysis of the gene transcription profile revealed downregulation of the cholesterol biosynthesis pathway. Inhibited cholesterol synthesis could impair viral budding, which, together with the reduced VZV DNA accumulation that occurs in infected neurons, provides a feasible mechanism for limited production of infectious virus in neurons and ultimately the establishment of latency.

Nicholas Baird, University of Colorado, presented data showing that interferon- $\gamma$ (IFN- $\gamma)$ inhibits VZV growth in human neurons. IFN- $\gamma$, produced during viral infection, stimulates transcription of genes that mediate antiviral responses. This laboratory tested whether IFN- $\gamma$ treatment of human neurons inhibits VZV gene expression in vitro. Infected neurons not treated with IFN- $\gamma$ developed a cytopathic effect in 4 weeks, during which time VZV DNA increased 7-fold and viral RNA accumulated. Infected neurons cultured in the presence of IFN- $\gamma$ for 8 weeks or infected neurons cultured in IFN- $\gamma$ for 4 weeks followed by cytokine removal for an additional 4 weeks had only a 2.8- and 3.6-fold increase of viral DNA, respectively, in the 8 weeks postinfection. Furthermore, levels of VZV transcripts did not increase between 4 
and 8 weeks postinfection when IFN- $\gamma$ was removed at 4 weeks postinfection, and even began to decrease when the cultures were maintained in IFN- $\gamma$ for the entire 8 weeks. In accordance with reduced DNA accumulation and mRNA levels when infected neurons were maintained in IFN- $\gamma$, less CPE was evident at 8 weeks postinfection compared to cultures which had IFN- $\gamma$ removed at 4 weeks postinfection. Replication of VZV DNA and transcription of viral genes was inhibited by IFN- $\gamma$, and the extent of virus gene expression in IFN- $\gamma$-treated neurons compared to VZV expression in latently infected human ganglia remains to be determined.

David Davido, University of Kansas, reported that during acute infection, HSV-1 alters cyclin-dependent kinase (CDK)-5 and its activating protein $\mathrm{p} 35$, which is required for efficient HSV-1 replication and plays a role in latency in murine trigeminal ganglia. During acute infection in neurons of mice, HSV-1 was found to increase p35 levels and change CDK-5 localization. Since CDK-5 is required for neuronal survival after activation of the DNA damage response, infected neurons stained positive for an early marker of this response, whereas markers of apoptosis were not readily detected. Thus, it is possible that HSV-1 upregulates expression of p35 to activate CDK-5 and inhibit apoptosis during infection following reactivation.

Paul Kinchington, University of Pittsburgh, presented new evidence for the role of inflammatory processes in the induction of pain in a rat model of postherpetic neuralgia (PHN) induced by VZV, and described HSV vector neuronal delivery as a novel strategy to relieve pain. PHN follows one third of zoster cases, lasts months to years, and is extremely debilitating. Importantly, most treatments do not provide complete relief. Live VZV inoculation of the rat footpad produces prolonged sensory hypersensitivity to mechanical and thermal stimuli like that seen in many PHN patients. Induction of pain requires VZV infectivity and is not a simple response to inoculated viral antigen. Evidence suggests that VZV infection of rat host tissues occurs but is abortive at a postentry stage and that neurons become infected from the peripheral inocula. While low numbers of neurons are involved and only VZV IE62 is detected, physiological changes occur that lead to peripheral neuronal retraction of axons from the skin. Affymatrix gene array analyses revealed upregulation of several inflammatory genes specific to painful dorsal root ganglia, including multiple members of the TNF receptor family. No strong CD4+ and CD8+ T cell infiltrate was seen like that encountered during the onset of HSV ganglionic latency. Since TNF activity is known to be involved in the stimulation of pain, therapy of VZV-induced pain was sought using replication-defective HSV-based neuron gene delivery of soluble TNF receptor. Excitingly, replication-defective HSV-mediated expression of sTNF-R at the ganglia alleviated much of the VZV-induced chronic pain indices for over 6 weeks, suggesting the promise of ganglionic expression of immune biologics for treatment of human PHN.
Robert Hendricks, University of Pittsburgh, described how reversible nerve damage regulates pathology in murine herpes simplex virus stromal keratitis (HSK). HSV-1 HSK is characterized by recurrent bouts of immunopathology triggered by viral reactivation from latency, resulting in corneal opacity, vascularization, and scarring. Nerve damage and loss of the corneal blink reflex (BR) accompanies HSK recurrences, but testing the BR is not usually part of the clinical HSK workup in humans or in mouse models. Although loss of the BR can lead to desiccation of the corneal surface, especially in mice that lack consensual BR, this possible contribution of nerve damage to HSK immunopathology has not been explored. This laboratory showed that chronic HSK in mice (maintained at least 70 days) is associated with loss of corneal BR. Preventing corneal desiccation through tarsorrhaphy (eyelid stitching) dramatically reduces HSK severity and resolves existing severe HSK in association with significant reduction in the neutrophil chemoattractant CXCL1. Corneas with resolved HSK exhibited residual attenuated blood vessels but complete corneal clarity or a slight haze with the clinical appearance of scar tissue, yet still without BR. Mice depleted of CD4+ T cells exhibited transient severe HSK, with concurrent resolution of HSK and recovery of the corneal BR and regeneration of corneal nerves. Thus, CD4-mediated chronic but reversible nerve damage and the accompanying corneal desiccation accounts for much of the inflammation associated with HSK in mice.

Benedikt Kaufer, Freie Universität Berlin, described an in vitro model for Marek's disease virus (MDV) latency. MDV is a highly oncogenic alphaherpesvirus that causes T cell lymphomas in chickens. In vivo, MDV replicates predominantly in B cells that transfer virus to T cells in which virus becomes latent. MDV is also able to transform CD4+ T cells, which spread and can develop into deadly lymphomas. Until now, no in vitro infection system for the target cells of MDV has been available due to the short-lived nature of $\mathrm{B}$ and $\mathrm{T}$ cells in vitro. Recently, cytokines and monoclonal antibodies that promote survival, proliferation, and activation of cultured chicken $\mathrm{B}$ and $\mathrm{T}$ lymphocytes were identified. These include CD40L and anti-TCR2 monoclonal antibodies, which were used to establish a culture system that allows productive infection of B and T cells with MDV in vitro. B cells isolated from the spleen, bursa, and blood were cultured in the presence of soluble CD40L and infected with fluorescently labeled MDV (UL47-RFP/Meq-GFP). Use of these productively infected B cells demonstrated that MDV can indeed be efficiently transferred from B cells to T cells. Stimulation with anti-TCR2 resulted in prolonged survival of uninfected T cells and allowed more efficient infection. Only a certain subset of $\mathrm{T}$ cells was latently infected, suggesting that MDV selectively infects T cells to become latent. Latently infected T cells could be maintained in culture for at least 5 weeks in the absence of TCR2 and ultimately resulted in the establishment of MDV- 
transformed lymphoblastoid cell lines in vitro. The immortalized cells have a phenotype comparable to that of MDVtransformed CD4 $+\alpha / \beta$ T cells in vivo. This novel model will allow analysis of latency in T cells as well as MDV-induced transformation of $\mathrm{T}$ cells in vitro.

Megan Steain, University of Sydney, discussed cell-typespecific modulation of anti-apoptotic proteins by VZV. VZV induces cell-type-specific apoptotic responses in human fibroblasts and MeWo cells, but not in T cells or neurons. VZV ORF63 confers resistance to apoptosis in neurons, although its mechanism of action remains unclear. This laboratory recently demonstrated that in ganglia from which VZV has reactivated, neuronal apoptosis is not prominent, as evidenced by a lack of cleaved caspase 3 staining despite an abundance of cytolytic cells. Thus, it appears that VZV can protect neurons from apoptosis induced by viral replication as well as by immune cells. Quantitative RT-PCR on mock- and VZV-infected fibroblasts for known anti-apoptotic genes revealed significant downregulation of X-linked inhibitor of apoptosis (XIAP), phosphoprotein enriched in astrocytes-15 (PEA-15), and cellular FLICE (FADD-like IL-1 $\beta$-converting enzyme)-inhibitory protein (c-FLIP) in VZV-infected fibroblasts compared to mock-infected cells at $24 \mathrm{~h}$ postinfection. Conversely, similar analysis of fetal dorsal root ganglionic explants indicated significant upregulation of cellular IAP2 (cIAP2) and PEA15 after VZV-infection compared to uninfected samples, while XIAP and cFLIP transcription was unchanged. Similar results were obtained using differentiated SH-SY5Y neuronallike cells. These changes may help explain cell-type-specific differences in apoptosis after VZV infection in fibroblasts and neurons. The ability of VZV to suppress apoptosis in neurons is likely to be a key step in the establishment of latency as well as during VZV reactivation to ensure that a viral reservoir is maintained.

Ilhem Messaoudi, University of California Riverside, presented data on robust mucosal immune responses in rhesus macaques infected intrabronchially with simian varicella virus (SVV), the non-human counterpart of VZV. While primary infection with VZV is thought to occur mostly through inhalation of virus-laden saliva droplets, the ensuing immune response in the lungs remains poorly understood. This laboratory recently showed that intrabronchial inoculation of young rhesus macaques with SVV recapitulates the hallmarks of acute and latent VZV infection in humans. In-depth analysis of the host immune response to acute SVV infection in the lungs revealed the following: a robust innate and adaptive immune response characterized by significant production of inflammatory cytokines and chemokines; an increased frequency of plasmacytoid dendritic cells that corresponded with an increase in IFN- $\alpha$ concentrations and vigorous $\mathrm{T}$ cell proliferation and differentiation especially within the CD8+ subset; and strong B cell proliferation and antibody production. Analysis of the peripheral immune response revealed kinetics similar to those observed in the lungs but with reduced magnitude. Interestingly, although the proliferative burst of CD4+ and CD8+ T cells exhibited similar kinetics and magnitude, a higher percentage of CD4+ T cells was SVV specific. The peak of CD4+ T cell response correlated with the cessation of viral replication in the lungs and resolution of viremia. Together with our previous studies using CD4+ T cell-depleted animals, these data suggest that a robust $\mathrm{CD} 4+\mathrm{T}$ cell response is required for the establishment of latency.

Joshua Schiffer, Fred Hutchinson Cancer Research Center, reported that HSV-2 shedding patterns in HIV-infected men suggest deficient cell-mediated immunity in both ganglia and genital mucosa. A signature feature of HIV infection is poor control of HSV-2 infection. While the mechanism underlying this observation is deficient CD4+ and CD8+ T cell function, it is unknown whether increased HSV-2 severity is due to poor cell-mediated immune control in neuronal sites of latency or in mucosal sites of lytic replication. While numerous animal and human studies have demonstrated that tissue resident $\mathrm{T}$ cells are important for immunosurveillance and rapid control of reactivation in both neurons and mucosa, the relative importance of immune control of latent versus lytic replication has not been estimated at the whole-organism level. Comparison of genital HSV-2 shedding patterns in $131 \mathrm{HIV}$-infected and 131 uninfected men who were matched according to other clinical characteristics revealed a higher frequency of HSV-2 genital shedding in HIV-positive men. Genital HSV-2 shedding is episodic in both groups, although HIV-infected men have higher shedding episodically and a longer mean duration of such episodes. These trends are increasingly evident in subsets of men with CD4+ T cells $<200 / \mathrm{uL}$. Early episode HSV-2 expansion rate, HSV-2 peak viral load, and viral clearance rate were equivalent in HIV-infected and uninfected patients and across all CD4+ counts. Longer episodes in HIV-infected persons were due to more frequent viral reexpansion that followed an initial phase of viral clearance. Application of a spatial, stochastic mathematical model to both data sets indicated a higher rate of HSV-2 release from neural tissue but a lower duration of mucosal cytolytic immune protection in HIV-infected patients. These results suggest that increased HSV-2 shedding in HIV-infected persons is due to poorer cellular immune function in both neurons (latent) and mucosa (lytic).

Travis Whitmer, Oregon Health and Science University, reported that SVV ORF61 inhibits NFKB signaling by preventing the ubiquitination of $\operatorname{IKB} \alpha$. Infection of rhesus macaques with SVV, the homolog of VZV, recapitulates many features of VZV pathogenesis and provides the opportunity to natively address issues, including the role of immune evasion strategies in VZV/SVV reactivation from latency. This issue is especially relevant since current VZV vaccines prevent zoster (shingles) in only about $50 \%$ of vaccinated individuals. With the ultimate goal of developing a more efficient VZV vaccine, 
this laboratory focused on identifying immune evasion mechanisms that influence spread and reactivation of VZV. Use of reporter cells showed that SVV inhibits TNF- $\alpha$-induced NFKB activation, and further studies revealed that the pathway is inhibited at the level of IKB $\alpha$ : In mock-infected cells, TNF- $\alpha$ induced IKB $\alpha$ phosphorylation and subsequent degradation, thereby activating the $\mathrm{NF} \kappa \mathrm{B}$ subunits, while neither phosphorylation nor degradation of $\mathrm{IKB} \alpha$ was observed in SVV-infected cells. Analogous to the reported involvement of VZV ORF61 in the inhibition of NFKB activation, SVV ORF61 also blocked NFKB activation through direct interaction with $\mathrm{I} \kappa \mathrm{B} \alpha$, preventing the protein's ubiquitination by the SCF complex and thus its degradation. In SVV-infected rhesus macaques, ORF61 is the most abundant transcript expressed during latency, most of which are antisense. ORF61 is not required for establishment of latency, but its requirement for reactivation is unclear. Identification of ORF61 regions required for evasion of NFKB signaling and construction of recombinant SVV lacking these regions are needed to elucidate the role of $\mathrm{NFKB}$ evasion in reactivation in vivo.

David Koelle, University of Washington, described how $\mathrm{CD} 8+$ and $\mathrm{CD} 4+\mathrm{T}$ cell responses to VZV cross-react with HSV. For HSV-1, both specific CD4+ and CD8+ T cells can be recovered from human trigeminal ganglia, despite little evidence for local viral protein expression. It is difficult to document VZV-specific T cells in latently infected dorsal root ganglia. Vaccination with live VZV reduces zoster via a mechanism that may involve T cells. Analyses to determine whether alphaherpesvirus-cross-reactive $\mathrm{T}$ cells play a role in control of VZV latency showed that $\mathrm{T}$ cells specific for several HSV-1 CD8+ T cell epitopes cross-recognize their VZV homolog and can kill VZV-infected HLA-A*0201-bearing cells, but not HLA-mismatched cells. About one third of bulk polyclonal VZV-reactive CD4+ cells cross-recognize HSV-1 when tested with lysates of virally infected cells that contain entire viral proteomes. Cross-reactive CD4 epitopes have also been defined. Thus, immunity to heterologous alphaherpesviruses such as HSV-1 may play a role in controling latent VZV infection and may modulate the efficacy of VZV vaccines.

Deepak Shukla, University of Illinois at Chicago, described the progressive loss of neuron size, number, and density in the trigeminal ganglia of mice with latent HSV-1 infection. Epidemiological studies suggest a link between HSV-1 infection and many chronic neurological diseases. HSV-1 infection of animal models, including mice, can result in behavioral and neurological changes, chronic pain, and inflammation. While neuronal loss in organotypic hippocampal slice cultures due to acute HSV-1 infection has been demonstrated, potential neurologic consequences of latent HSV-1 infection remain poorly understood. To determine whether latent HSV-1 infection can cause neuronal insult in mice, the corneas of adult BALB/c mice were infected with HSV-1 for 1, 12, or 31 weeks and infection was allowed to spread to the trigeminal ganglia. At each time point, the trigeminal ganglia of infected mice were examined by immunohistochemistry for productive or latent HSV-1 infection, inflammation, oxidative damage, and any associated changes in neuron size, density, and number. Results indicate that latent HSV-1 infection between 12 and 31 weeks was associated with chronic inflammation, oxidative damage, and progressive insult to trigeminal ganglia neurons. Infected trigeminal ganglia showed lower mean neuron density and number as well as lower neuron and neuronal nucleus diameters, but higher oxidative damage relative to mock-infected controls. The extent of neuronal injury correlated directly with the extent of inflammation and oxidative radicals in the trigeminal ganglia of infected animals. Overall, latent HSV infection was associated with detectable neuronal injury, which may contribute to development of chronic neurological diseases, including Alzheimer's disease, autism, and schizophrenia.

Martine Aubert, Fred Hutchinson Cancer Research Center, described inactivation of latent HSV-1 by homing endonuclease (HE)-directed mutagenesis. Previously, this laboratory reported the use of rare-cutting HE for targeted mutagenesis of essential HSV genes as a therapeutic approach to cure chronic/ latent viral infections. In a model of latent HSV-1 infection, in situ targeted mutagenesis of latent HSV genomes was demonstrated in fibroblasts exposed to both HSV-specific HE (HSVHE) and Trex2, a $3^{\prime}$ to $5^{\prime}$ DNA exonuclease. While HE treatment resulted in a significant decrease in infectious virus production after reactivation, the mutation frequency within latent genomes remained modest, possibly due to their compact state driven by epigenetic control mechanisms and resulting low accessibility. To test whether HSV-1 DNA conformation might impact HE-mediated mutagenesis, HSV-1-infected cells were exposed to HSV-HE in the presence of either trichostatin A (TSA), a histone deacetylase inhibitor (HDACi) that induces derepression of the viral episome, or IFN- $\alpha$ to increase viral genome repression. The mutation frequency in HE-treated cells exposed to TSA was increased 2-fold, while HE-mediated mutagenesis in HE-treated cells exposed to IFN- $\alpha$ was inhibited compared to HE-treated cells in the absence of either drug. Initial experiments in primary murine neurons showed that active HSV replication is decreased 2-fold in cells treated with HSV-HE in the presence of Trex2, and HE target site amplicon analysis revealed the presence of mutations in HSV genomes. Efforts to test whether HSV genomes in latently infected neurons can be successfully targeted for mutagenesis and whether treatment with TSA or other HDACi can improve mutagenesis efficiency are ongoing, with the ultimate goal of curing HSV infection in latently infected neurons in vivo.

Edouard Cantin, Beckman Research Institute of City of Hope, discussed a HSV-1 gene required specifically for reactivation from latency. Reactivation of latent HSV-1 accounts 
for recurrent disease and widespread virus dissemination. In the absence of a vaccine, the best prospect of developing one rests in unraveling the mechanisms controlling reactivation. This laboratory identified a HSV-1 strain with an impaired reactivation phenotype during characterization of two HSV-1 strain $17+$ viruses (HSV-Crel and a Cre reporter strain, HSV- $\beta$-gal-OFF, abbreviated as HSV- $\beta$-gal) in vitro and in vivo. Although replication of both strains was equivalent in vitro and in vivo in peripheral mouse tissues, HSV-Crel did not reactivate in ex vivo trigeminal ganglia explant cultures. A second independently isolated HSV-1-Cre virus had the same phenotype, suggesting that the mutation was specific and not due to a random homologous recombination event during isolation of the Cre virus. An YFP Cre rescuant was also reactivation-impaired, excluding Cre cellular toxicity as a reason for the phenotype. Mixed infections at different ratios in which HSV-Cre was found to block reactivation of HSV- $\beta$ gal in trans suggested that the mutation(s) was due to Cre expression promoting recognition of a cryptic loxP site(s) in a gene(s) specifically required for reactivation. To identify the involved gene(s), HSV-Cre1, HSV-Cre2, and HSV- $\beta$-gal were sequenced on the Illumina platform and the assembled genome sequences were compared to the reference HSV-1 $17+$ and to each other. Annotation of mutated genes conserved in HSV-Cre1 and HSV-Cre2, but not in HSV- $\beta$-gal, identified reference $17+$ strain RL2, which encoded ICP0, as a highpriority candidate gene responsible for the phenotype. Current studies are focused on testing whether the mutation in RL2 effectively separates the functions of ICP0 required for replication from those required for reactivation.

Anna Cliffe, Neuroscience Center, UNC Chapel Hill, reported that c-Jun $\mathrm{N}$ terminal kinase (JNK) activity is required for initiation of HSV lytic gene expression during reactivation from latency. During latency in ganglion neurons, lytic HSV genes are silenced and assembled into heterochromatin, but the mechanism by which lytic gene expression initiates from silenced genomes is not understood. Known HSV reactivation stimuli include NGF deprivation, phosphatidylinositide 3kinase (PI3K) inhibition, and dexamethasone treatment, all of which activate JNK. In a primary neuronal system of HSV latency, reactivation induced by $\mathrm{PI} 3 \mathrm{~K}$ inhibition and dexamethasone was blocked by the addition of the JNK inhibitors. Neither PI3K inhibition, NGF deprivation, nor dexamethasone treatment induced release of cytochrome $\mathrm{c}$ from mitochondria or neuronal cell death at postnatal day 18 , indicating that reactivation was not a consequence of apoptosis. Inhibition of the JNK signaling pathway prevented phase I of HSV lytic gene expression, which is thought to involve an initial burst of gene expression occurring independently of VP16, and both JNK and c-Jun were enriched on viral lytic promoters during phase I. These results identify a key role for the JNK signaling pathway in mediating the initial induction of lytic gene expression during HSV reactivation.
Leigh Zerboni, Stanford University Medical Center, described investigations of VZV neurotropism using the SCIDdorsal root ganglia mouse model. Postherpetic neuralgia (PHN) after reactivation of latent VZV in ganglionic neurons resulting in zoster is a leading cause of intractable neuralgic pain, including allodynia (pain after a light touch). Risk factors for development of PHN include age, prodromal symptoms before zoster, and rash severity. The pathophysiology of PHN-related tactile allodynia is not well understood. Analysis of VZV neurotropism in a SCID-dorsal root ganglia mouse model has revealed a subtype-specific restriction for replication in mechanoreceptive neurons, identified by RT97 immunoreactivity. While VZV particle entry and immediate-early (IE) protein nuclear expression were unimpaired, events in VZV replication that follow viral DNA synthesis, such as cytoplasmic accumulation of IE62 and IE63, were blocked. VZV lytic replication spread to satellite glial cells that encapsulate mechanoreceptive neurons and are critical for neuronal survival. Loss of supporting satellite cells results in selective loss of the RT97 immunoreactive neuronal subpopulation when examined at 56-70 days after infection. Thus, longlasting PHN-related tactile allodynic pain appears to result from axonopathy in cutaneous mechanoreceptive neurons that have lost or impaired satellite cell support due to ganglionic spread of VZV during reactivation.

Joshua Geltz, Southern Illinois University School of Medicine, reported that HSV-2 glycoproteins are not the dominant antigens of a latency-biased HSV-2 ICP0-mutant vaccine. Infected cell protein 0 (ICP0) is a master regulator of the balance between latency and replication in HSV. When ICP0 fails to accumulate, low multiplicities of HSV-2 ICP0-mutant viruses establish quiescent infections in $99 \%$ of virus-infected cells. Likewise, ICP0 is required for efficient HSV-1 reactivation, and ectopic expression of ICP0 is sufficient to trigger HSV-1 reactivation. Analysis of the potential of latencybiased HSV-2 ICP0 viruses to serve as a safe and effective live HSV-2 vaccine showed that HSV-2 ICP0 viruses, such as HSV-2 $0 \Delta$ NLS, are profoundly IFN sensitive and avirulent in vivo and elicit superior protection against genital herpes, consistent with a 50-fold higher IgG antibody response to HSV-2 antigen than that elicited by a glycoprotein D (gD) subunit vaccine. Based on the correlation between virusspecific IgG and protection, studies were initiated to explore which of HSV-2's 75 proteins were the most dominant IgG antibody generators (antigens) of the HSV-2 $0 \Delta$ NLS vaccine. Western blots indicated that the $0 \Delta$ NLS vaccine generated an IgG response against $>10 \mathrm{HSV}-2$ proteins. Surprisingly, the $56-\mathrm{kDa} \mathrm{gD}$ protein was not among the most dominant antigens, and instead, virus-specific IgG heavily targeted viral proteins $>100 \mathrm{kDa}$ in size and abundant in virus-infected cells but scarce in virions. Immunoprecipitation of HSV-2 proteins with $0 \Delta$ NLS antiserum followed by mass spectrometry revealed that ICP10 and ICP8 accounted for $40 \%$ of viral 
peptides in immunoprecipitates. These results raise questions about the assumption that HSV-2 glycoproteins should be the focal point of vaccine development efforts, and highlight the primary advantage of a live HSV-2 vaccine, which may prevent HSV-2 genital herpes today rather than awaiting identification of which antigen(s) elicits the protective immune response.

Michael Gershon, Columbia University, discussed enteric zoster in humans and guinea pigs. This laboratory found that VZV infects the human gut and becomes latent in neurons of the enteric nervous system (ENS). Although VZV is not present in the ENS at birth, latent infection in adults is extremely prevalent and may include almost all individuals who have experienced varicella or who have been vaccinated. Transcripts encoding VZV ORF63 are found only in segments of gut that contain ganglia; in two patients with Hirschsprung's disease, these transcripts were detected in proximal segments of excised colon containing enteric ganglia, but not in distal aganglionic segments of bowel. VZV reactivates from ganglionic neurons to cause zoster (shingles) and also likely reactivates from enteric neurons, although the consequences of such occurrences are not well known. The association of VZV with pseudo-obstruction in Ogilvie's syndrome suggests that enteric zoster may be life-threatening. This laboratory recently documented that VZV reactivation caused an episode of severe gastric hemorrhage necessitating a partial gastrectomy in a 16-year-old immunocompetent boy. DNA encoding vaccine-type VZV genes (ORFs 40 and 67) was detected in saliva and tissue removed at surgery; moreover, the immunoreactivities of VZV proteins ( $\mathrm{gE}$ and ORF63p) were found in epithelial cells at the margins of the lesions. No VZV gene products were found in repeat biopsies of the stomach after recovery. To further explore enteric VZV infection and its consequences, a guinea pig model was developed. Despite the human species restriction of VZV infection, guinea pig $\mathrm{T}$ lymphocytes cocultured with VZV-infected human embryonic lung fibroblasts became infected. Infected lymphocytes expressed immediate-early (IE) (ORF62p) and late VZV proteins (gE), and electron microscopic examination revealed virions produced by infected lymphocytes. As in VZV-infected human cells, infection of guinea pig lymphocytes was cell-associated, and filterable virus was not secreted. When VZV-infected guinea pig $\mathrm{T}$ cells were injected into recipient guinea pigs, VZV transcripts and gene products (IE and late genes) were detected within 2 days in resident macrophages of liver, lung, and spleen. After 4 weeks, VZV disappeared from these organs but was found in the ENS and dorsal root ganglia, where only IE genes were then transcribed. Latent VZV within enteric neurons was confirmed by in situ hybridization to detect transcripts and by immunocytochemistry to detect IE proteins. Repeat experiments with GFP-tagged VZV under the ORF66 promoter revealed that intravenous administration of VZV-infected lymphocytes leads to latency in virtually all enteric neurons and most dorsal root ganglia. Preliminary evidence suggests that corticotrophin-releasing hormone, which mediates effects of stress, reactivates VZV in the ENS and causes VZV DNA to appear in guinea pig saliva. The guinea pig will be exploited to investigate stimuli that provoke VZV reactivation in vivo, and to determine the pathophysiology of disorders arising from enteric zoster, which may be occult in humans since it occurs without rash.

Jürgen Haas, University of Edinburgh, presented work on novel host protein interaction partners of latent VZV proteins which have been detected by a genome-wide yeast two-hybrid screen. This laboratory cloned a set of 103 VZV gene constructs and tested them against a human brain library and a normalized library of 12,381 human cDNA clones. A total of 876 interactions between $61 \mathrm{VZV}$ and 755 human proteins were identified, including interactions of ORFs 21, 29, 40, 62, and 63 which are expressed in latently infected neurons. The interaction of ORF21 with a neuronal kinase and a signaling molecule of the NF-kB pathway, as well as the interaction of ORF62 with histone and actin proteins, may provide some clues on previously unknown functional activities of these proteins during the latent stage of infection.

Karen Johnson, Rosalind Franklin University of Medicine and Science, reported that the host nuclear innate immune sensor IFI16 restricts HSV-1 replication by regulating gene expression of all temporal classes of HSV-1 genes and by inducing chromatin remodeling. IFI16 is a multifunctional, predominantly nuclear protein that is involved in transcriptional regulation and innate immune signaling. IFI16 is constitutively expressed in most cell types, including fibroblasts, epithelial cells, and neurons, and can be induced by various cell stressors, including viral infection. During the establishment of latent HSV-1 infection in ganglia, expression of all HSV-1 genes except for the LAT transcript is repressed. The mechanisms of this repression are unclear, although chromatin modification has a known role in maintaining latency. HSV-1 causes specific degradation of IFI16 at later times during lytic infection dependent on the viral E3 ubiquitin ligase ICP0, suggesting that IFI16 exerts negative pressure on HSV-1 replication. Indeed, IFI16 has been described as a restriction factor for human CMV and HSV-1 replication, although mechanisms of HSV-1 restriction were not investigated. This laboratory's analysis in multiple cell types shows that IFI16 has a profound negative effect on HSV-1 replication and expression of all temporal classes of HSV-1 genes. Through CRISPR technology, a cell line was established with a permanent lesion in the IFI16 coding sequence, resulting in an effective permanent IFI16-negative cell line. Analysis of these cells and the parental IFI16-positive cells showed that IFI16 binds HSV-1 genomic DNA and inhibits association of RNA polymerase II and transcription factors with HSV-1 transcription start sites. IFI16 also caused a global shift in chromatin 
modification; total histone $\mathrm{H} 3$ association with viral and cellular promoters was unchanged, but IFI16 depletion resulted in increased markers of active chromatin and decreased markers of repressive chromatin associated with both viral and cellular promoters. These studies suggest a potential role for IFI16 in repression of lytic HSV-1 gene expression during the establishment of latency.

Ian Mohr, New York University School of Medicine, discussed regulation of HSV-1 latency by signaling through the neuronal DNA damage response (DDR) network. While genotoxic stress triggers a complex DDR signaling network, its impact on neurons and the neurotrophic viruses that colonize them remains poorly understood. Environmental insults and normal neuronal activity are a continuous source of double-stranded DNA (dsDNA) breaks, necessitating an effective DDR to preserve genomic integrity and maintain homeostasis throughout the lifetime of the neuron. This laboratory investigated the ability of the DDR to control HSV-1 latency by exploiting a reconstituted system in which latent infections are established in homogeneous cultures of rat primary neurons and which recapitulates key biological hallmarks of HSV-1 latency and reactivation. Regulation of dsDNA breaks was found to be a potent inducer of HSV-1 reactivation. Surprisingly, suppression of host dsDNA break repair pathways in the absence of exogenous DNA-damaging agents stimulated reactivation without producing detectable dsDNA breaks. Furthermore, interference with DDR signaling markedly reduced activation of Akt, a nodal point in NGFmediated signaling needed to suppress reactivation. Thus, continual signaling through the DDR network is required to maintain HSV-1 latency in cultured neurons and provides a mechanism whereby the genomic integrity of the host neuron can be sampled by neurotrophic pathogens.

Farhana Musarrat, Louisiana State University Health Sciences Center, described CTCF as a potential mediator in the latent-lytic switch in HSV-1 infection. Seven occupied CTCF binding motifs have been identified in the latent HSV-1 genome and are positioned in an orientation that flanks the reactivation-critical LAT and each immediate-early region separately. This laboratory found that at least three of the HSV-1 CTCF binding motifs are occupied by CTCF during latency in a transcript-dependent manner and that these three sites undergo CTCF eviction at early times postreactivation in mice latently infected with 17Syn+. These findings support the possibility that each CTCF domain in HSV-1 is independently regulated. ChIP assays to examine the CTCF occupation of the remaining CTCF binding motifs of HSV-1 during latency and after reactivation revealed significant enrichment of one site in particular, the CTRL1 site upstream of the LAT promoter, in CTCF during latency relative to other CTCF motifs in wild-type HSV-1 ( $>$ 3-fold, $p<0.05$ ). After explantinduced reactivation, the CTRL1 domain retained CTCF occupancy in wild-type HSV-1. Thus, the CTRL1 motif may play a regulatory role in chromatin domains in HSV-1 not only during latency but also after reactivation. ChIP assays also revealed enrichment of the CTRS1/2 motif located downstream of the ICP4 region, in CTCF during latency, but eviction of CTCF from this motif after explant reactivation was delayed in wild-type virus. Subsequent transient transfection assays showed that the CTRS $1 / 2$ motif functions as an enhancer-blocker to the LAT enhancer. Finally, chromosome conformation capture assay of latently infected mouse trigeminal ganglia indicated that genomic sequences near the CTRL1 motif and the CTRS1/2 motifs in HSV-1 are proximally ligated, suggesting that the CTRL1 and CTRS1/2 regions of HSV-1 may be involved in formation of a CTCFmediated intrachromosomal loop to regulate immediate-early gene transcription during latency.

Alexander Rowe, University of Pittsburgh, described the sequestration of memory $\mathrm{T}$ cells in latently infected ganglia. HSV-1 latency in the trigeminal ganglion is maintained in part by a resident $C D 8+T$ cell population established by infiltration from the blood during acute infection. Studies by this laboratory have shown that increases in this $\mathrm{CD} 8+$ population during latency ( $>30$ days postinfection), either as recovery from stress events or in response to subsequent immune challenges, are entirely independent of peripheral $\mathrm{T}$ cells. This exclusively HSV-1-specific CD8+ population cannot be augmented by adoptive cell transfer of HSV-1-specific CD8+ T cells or by therapeutic vaccination, i.e., the door to the TG is closed to CD8+ T cells during HSV-1 latency. Efforts to understand the molecular machinery that opens and closes this door initially focused on the kinetics of CD8+ T cell entry into the TG. In C57Bl/6 mice infected with KOS, adoptively transferred congenically marked HSV-1-specific CD8+ effector $T$ cells could not enter trigeminal ganglia beyond 17 days after infection. Time course analysis of the trigeminal ganglia transcriptome by nanostring chip assay revealed that this door closure is directly preceded by a decrease in expression of chemokines CXCL10, CXCL9, and CCL5. Moreover, expression of these chemokines was significantly reduced when infected mice were depleted of CD4+ T cells. The tendency of HSV-specific CD4+ T cells to be migratory, while their CD8+ counterparts tend to become sequestered in infected tissue, raises the possibility of indirectly augmenting the size of the $\mathrm{CD} 8+\mathrm{T}$ cell population in latently infected trigeminal ganglia through vaccines designed to activate $\mathrm{CD} 4+\mathrm{T}$ cells.

Patrick Stuart, Saint Louis University, discussed CD28 costimulation as a requirement for optimal antigen-specific $\mathrm{CD} 8+\mathrm{T}$ cell expression during lytic infection. Mice are very resistant to herpetic reactivation, which results in shedding of virus at the periphery. In a recurrent model of herpetic stromal keratitis, two strains of mice showed measurable shedding of virus from the cornea in the absence of a reactivation signal. Mice deficient in CD28 demonstrated a greater frequency of "spontaneous" reactivation (10-20\%). Two separate 
hypotheses were tested to examine the mechanism underlying these observations. First, the longer virus shedding duration from the cornea after reactivation in CD28KO mice (12 vs 6 days for wild-type mice) raised the possibility that impaired control of virus production results in a more pronounced infection of trigeminal ganglia. Both RT-PCR analysis to measure the total number of genomes in infected trigeminal ganglia and FISH analysis to detect numbers of neurons expressing LAT revealed slightly more genomes and infected cells in CD28KO mice, but the difference was not significant. Second, this laboratory tested whether antigen-specific $\mathrm{CD} 8+\mathrm{T}$ cell responses are impaired in CD28KO mice and thus control of latency is not robust. Results revealed fewer numbers of CD8+ T cells isolated from $\mathrm{CD} 28 \mathrm{KO}$ mice than from wild-type mice and a significantly lower percent of CD8 $+\mathrm{T}$ cells expressing the immunodominant $\mathrm{T}$ cell receptor in $\mathrm{CD} 28 \mathrm{KO}$ mice than in wild-type mice (30 vs $50 \%$ ) at day 30 . Further studies will address subdominant $\mathrm{CD} 8+\mathrm{T}$ cells recently shown to be important in maintaining latency. Overall, the data support the notion that the primary mechanism for spontaneous reactivation in CD28KO mice is inefficient expansion and migration of antigen-specific $\mathrm{CD} 8+\mathrm{T}$ cells to the trigeminal ganglia.

Acknowledgments This work was supported in part by Public Health Service grant AG04651 (R.C.) from the National Institutes of Health. The Colorado Alphaherpesvirus Latency Society thanks abcam, Addgene, BioRad Laboratories, Cellular Dynamics, Diagenode, Fisher Scientific, Don Gilden, Integrated DNA Technologies, Kiwanis Club of DTC Foundation, Light Labs, Macherey-Nagel Inc., Rocky Mountain Scientific Instrument Maintenance LLC, PerkinElmer, Quanta Biosciences, and the National Shingles Foundation for their generous support. We especially thank the owners of the Christiania Lodge at Vail. We thank Marina Hoffman for editorial assistance and Cathy Allen for preparing the manuscript.

Conflict of interest The authors declare that they do not have any conflict of interest. 